\title{
Scale Invariance in the Spectral Action
}

\author{
Ali H. Chamseddine ${ }^{1}$, Alain Connes ${ }^{2,3,4}$ \\ ${ }^{1}$ Center for Advanced Mathematical Sciences and \\ Physics Department, American University of Beirut, Lebanon. \\ ${ }^{2}$ College de France, 3 rue Ulm, F-75005, Paris, France \\ ${ }^{3}$ I.H.E.S. Bures-sur-Yvette, France \\ ${ }^{4}$ Vanderbilt University, Tennessee USA.
}

\begin{abstract}
The arbitrary mass scale in the spectral action for the Dirac operator in the spectral action is made dynamical by introducing a dilaton field. We evaluate all the low-energy terms in the spectral action and determine the dilaton couplings. These results are applied to the spectral action of the noncommutative space defined by the standard model. We show that the effective action for all matter couplings is scale invariant, except for the dilaton kinetic term and Einstein-Hilbert term. The resulting action is almost identical to the one proposed for making the standard model scale invariant as well as the model for extended inflation and has the same low-energy limit as the Randall-Sundrum model. Remarkably, all desirable features with correct signs for the relevant terms are obtained uniquely and without any fine tuning.
\end{abstract}




\section{Introduction}

It is known that the standard model of strong and electroweak interactions is classically almost scale invariant, and that the only terms that break the dilatation symmetry are the mass terms in the Higgs sector. Scale invariance of the classical Lagrangian can be achieved by introducing a compensating dilaton field [1], [2], [3]. Breaking of scale invariance occurs after the electroweak symmetry is broken spontaneously through the generation of radiative corrections to the scalar potential. The dilaton mass scale is much larger than the weak scale and could be as large as the GUT scale or Planck scale. This leads naturally to consider the coupling of the dilaton to gravity. The dilaton is always part of the low energy spectrum in string theory. Historically it first appeared in the Jordan-Brans-Dicke theory of gravity which corresponds to one particular coupling of the dilaton to the metric [2]. The dilaton plays a fundamental role in models of inflation [4]. It also appears in the gravitational couplings of the noncommutative Connes-Lott formulation of the standard model [5], [6],[7], [8], where the dilaton is the scalar field that couples the two sheets of space-time. The resulting matter interactions in this case are also scale invariant, and the gravitational couplings are different than the Jordan-Brans-Dicke theory. More recently, a scalar field, the radion field, appeared in the Randall-Sundrum scenario (RS) of compactification [9] which is related to the question of masses and scales in physics. The RS scenario was shown to be equivalent to the results derived from noncommutative geometry, [10], which is not too surprising, because both the Connes-Lott model and the RS model, describe a system with two branes.

At present, and within the noncommutative geometric picture, the spectral action gives the most elegant formulation of the standard model [11], [12]. All details of the standard model as well as its unification with gravity are achieved by postulating the action

$$
\text { Trace } F\left(D^{2} / m^{2}\right)+\langle\Psi|D| \Psi\rangle
$$

where $D$ is the Dirac operator of a certain noncommutative space and $\Psi$ is a spinor in the Hilbert space of the observed quarks and leptons. However, the dilaton field does not appear in the spectral action, which is to be contrasted with the Connes-Lott formulation of the noncommutative action where the dilaton field is part of the gravitational interactions. This suggests that 
the Dirac operator used in the construction of the spectral action should be modified in order to take into account the presence of the dilaton.

The appearance of the dilaton field in physical models is related to the question of mass and scales. It is therefore natural to consider replacing the mass parameter in the ratio $D^{2} / \mathrm{m}^{2}$ by a function of the dilaton, thus introducing a sliding scale, as the Dirac operator have the dimension of mass. This is also relevant when dealing with non-compact manifolds where $D$ no longer has discrete spectrum and the counting of eigenvalues requires a localization. Let the dynamical scale factor $\rho$ be written in the form

$$
\rho=m e^{\phi}
$$

where we assume that $\phi$ is dimensionless. The dilaton can be related to a scalar field $\sigma$ of dimension one by writing

$$
\phi=\frac{1}{f} \sigma,
$$

where $f$ is the dilaton decay constant. The mass scale $m$ can be absorbed by the redefinition

$$
\phi \rightarrow \phi-\ln m,
$$

and therefore we can assume, without any loss in generality, that $\rho=e^{\phi}$. One can always recover the scale $m$ by performing the opposite transformation $\phi \rightarrow \phi+\ln m$. Now using $\rho$ instead of the scalar $m$ in the counting of eigenvalues :

$$
N(m)=\operatorname{Dim}\left\{D^{2} \leq m^{2}\right\} \rightarrow N(\rho)=\operatorname{Dim}\left\{D^{2} \leq \rho^{2}\right\}
$$

is equivalent to replacing the operator $D^{2} / m^{2}$ in [11] by

$$
P=e^{-\phi} D^{2} e^{-\phi} \text {. }
$$

If we insist that the metric $g_{\mu \nu}$ be dimensionless to insure that its flat limit be the Minkowski metric, then the scale $m$ will explicitly appear in the action after rescaling $e^{\phi} \rightarrow m e^{\phi}$. Otherwise we can absorb this mass scale by assuming that the metric has the dimension of mass.

The aim of this paper is to determine the interactions of the dilaton field $\phi$ with all other fields present in the spectral action formulation of the standard model. Because of the spectral character of the action, it is completely 
determined from the form of $P$ and there is no room for fine tuning the results. It is then very reassuring to find that the resulting interactions are identical to those constructed in the literature by postulating a hidden scale invariance of the matter interactions [3]. These are also equivalent to the interactions of the radion field in the RS model [9]. All of these results now support the conclusion that space-time at high-energies reveals its discrete structure, and is governed by noncommutative geometry. The plan of this paper is as follows. In section two we briefly review the derivation of the spectral action and comment on the modifications needed to include the dilaton. In section three we derive the Seeley-de Witt coefficients of the spectral action in presence of the dilaton. In section four we give the full low-energy spectral action including dilaton interactions specialized to the noncommutative space of the standard model. In section five we compare our results with those obtained by imposing scale invariance on the standard model interactions, to the RS model and the model of extended inflation. Section six is the conclusion. The appendices contain detailed proofs of some identities used.

\section{A summary of spectral action}

We begin by summarizing the results of [11]. The square of the Dirac operator appearing in the spectral triple of a noncommutative space is written in the following form suitable to apply the standard local formulas for the heat expansion (see [13] section 4.8).

$$
D^{2}=-\left(g^{\mu \nu} I \partial_{\mu} \partial_{\nu}+A^{\mu} \partial_{\mu}+B\right),
$$

where $g^{\mu \nu}$ plays the role of the inverse metric, $I$ is the unit matrix, $A^{\mu}$ and $B$ are matrix functions computed from the Dirac operator. The bosonic part of the spectral action can be expanded in a power series as function of the inverse scale $m$, and is given in dimension 4 by

$$
\operatorname{Trace}\left(F\left(D^{2} / m^{2}\right)\right) \simeq \sum_{n \geq 0} f_{n} a_{n}\left(D^{2} / m^{2}\right),
$$


where $F$ is a positive function and

$$
\begin{aligned}
f_{0} & =\int_{0}^{\infty} F(u) u d u, \quad f_{2}=\int_{0}^{\infty} F(u) d u, \\
f_{2 n+4} & =(-1)^{n} F^{(n)}(0), \quad n \geq 0 .
\end{aligned}
$$

The positivity of the function $F$ will insure that the actions for gravity, YangMills, Higgs couplings are all positive and the Higgs mass term is negative. We will comment on the positive sign of the cosmological constant at the end of the paper.

The first few Seeley-deWitt coefficients $a_{n}\left(D^{2} / m^{2}\right)$ are given (see [13] Theorem 4.8) by $^{1}$

$$
\begin{aligned}
a_{0}\left(D^{2} / m^{2}\right)= & \frac{m^{4}}{16 \pi^{2}} \int_{M} d^{4} x \sqrt{g} \operatorname{Tr}(1) \\
a_{2}\left(D^{2} / m^{2}\right)= & \frac{m^{2}}{16 \pi^{2}} \int_{M} d^{4} x \sqrt{g} \operatorname{Tr}\left(-\frac{R}{6}+E\right) \\
a_{4}\left(D^{2} / m^{2}\right)= & \frac{1}{16 \pi^{2}} \frac{1}{360} \int_{M} d^{4} x \sqrt{g} \operatorname{Tr}\left(-12 R_{; \mu}^{\mu}+5 R^{2}-2 R_{\mu \nu} R^{\mu \nu}\right. \\
& \left.+2 R_{\mu \nu \rho \sigma} R^{\mu \nu \rho \sigma}-60 R E+180 E^{2}+60 E_{; \mu}{ }^{\mu}+30 \Omega_{\mu \nu} \Omega^{\mu \nu}\right)
\end{aligned}
$$

while the odd ones all vanish

$$
a_{2 n+1}\left(D^{2} / m^{2}\right)=0 .
$$

The notations are as follows, one lets $\Gamma_{\mu \nu}^{\rho}(g)$ be the Christoffel symbols of the Levi-Civita connection of the metric $g$ and lets

$$
\Gamma^{\rho}(g)=g^{\mu \nu} \Gamma_{\mu \nu}^{\rho}(g)
$$

The connection form $\bar{\omega}$, its curvature $\Omega$, and the endomorphism $E$ are then defined by (see [13] section 4.8)

$$
\begin{aligned}
\bar{\omega}_{\mu} & =\frac{1}{2} g_{\mu \nu}\left(A^{\nu}+\Gamma^{\nu}(g) I\right), \\
\Omega_{\mu \nu} & =\partial_{\mu} \bar{\omega}_{\nu}-\partial_{\nu} \bar{\omega}_{\mu}+\left[\bar{\omega}_{\mu}, \bar{\omega}_{\nu}\right], \\
E & =B-g^{\mu \nu}\left(\partial_{\mu} \bar{\omega}_{\nu}+\bar{\omega}_{\mu} \bar{\omega}_{\nu}-\Gamma_{\mu \nu}^{\rho}(g) \bar{\omega}_{\rho}\right) .
\end{aligned}
$$

\footnotetext{
${ }^{1}$ According to our notations the scalar curvature $R$ is negative for spheres (see [13] section 2.3) and the space is Euclidean.
} 
To understand algebraically the dependence in the operator $D$ it is convenient to express the above coefficients as residues and this is done as follows in the generality that we need. One lets $P$ be a second order elliptic operator with positive scalar principal symbol and defines a zeta function as

$$
\zeta_{P}(s)=\operatorname{Trace}\left(P^{-s / 2}\right)
$$

One then gets in the required generality for our purpose the equality

$$
\begin{aligned}
\operatorname{Trace}\left(F\left(P / m^{2}\right)\right) & \sim \frac{m^{4}}{2} \operatorname{Res}_{s=4} \zeta_{P}(s) f_{0} \\
& +\frac{m^{2}}{2} \operatorname{Res}_{s=2} \zeta_{P}(s) f_{2}+\zeta_{P}(0) f_{4}+\cdots
\end{aligned}
$$

which, using the Wodzicki residue which is given independently of $D$ by

$$
f T=\operatorname{Res}_{s=0} \operatorname{Trace}\left(T\left(D^{2}\right)^{-s / 2}\right)
$$

can be written as

$$
\begin{aligned}
\operatorname{Trace}\left(F\left(P / m^{2}\right)\right) & \sim \frac{m^{4}}{2} f_{0} f P^{-2} \\
& +\frac{m^{2}}{2} f_{2} f P^{-1}+f_{4} \zeta_{P}(0)+\cdots .
\end{aligned}
$$

We want to compute the spectral action associated with the operator $P=$ $e^{-\phi} D^{2} e^{-\phi}$ i. e. to determine the dependence of the spectral action on the dilaton field $\phi$. The first term $f P^{-2}$ is a Dixmier trace and one can permute the functions with the operators without altering the result since the Dixmier trace vanishes on operators of order $<-4$. One thus gets, for any test function $h$,

$$
f h P^{-2}=f h e^{4 \phi} D^{-4}
$$

which shows that we get an overall factor of $e^{4 \phi}$ multiplying $a_{0}\left(x, D^{2}\right)$. For the second term $f P^{-1}$ we have

$$
f h P^{-1}=f h e^{\phi} D^{-2} e^{\phi}=f h e^{2 \phi} D^{-2}
$$

using the trace property of the residue and again we get an overall factor of $e^{2 \phi}$ multiplying $a_{2}\left(x, D^{2}\right)$. Note that the result remains valid when the test 
function $h$ is taken with values in endomorphisms of the vector bundle on which $P$ is acting. This suggests that the invariance of the $a_{2}$ term (up to the $e^{2 \phi}$ scale factor) takes place before taking the fiberwise trace. The direct computation below in equation (18) will confirm this point .

The term $f_{4} \zeta_{P}(0)$ is more tricky to analyze and we shall only give now a heuristic argument explaining why it should be independent of $\phi$. We shall then check it by a direct calculation. The formal argument proceeds as follows. First one lets

$$
P(t)=e^{-t \phi} D^{2} e^{-t \phi}
$$

so that $P(0)=D^{2}$ and $P(1)=P$ with the above notations. Let then

$$
Y(t)=\log P(t)-\log P(0) .
$$

Using the equality $(a>0)$

$$
\log a=\int_{0}^{\infty}\left(\frac{1}{\lambda+1}-\frac{1}{\lambda+a}\right) d \lambda
$$

applied to $P(t)$ one obtains the relation

$$
\frac{d}{d t} Y(t)=-\int_{0}^{\infty}(P(t)+\lambda)^{-1}(\phi P(t)+P(t) \phi)(P(t)+\lambda)^{-1} d \lambda .
$$

One then shows that

$$
\frac{d}{d t} Y(t)=-2 \phi+[P(t), C(t)]
$$

where $C(t)$ is a pseudodifferential operator (see appendix A). Thus one gets a similar expression

$$
Y=Y(1)=-2 \phi+\int_{0}^{1}[P(t), C(t)] d t
$$

Next one uses the expansional formula

$$
e^{A+B} e^{-A}=\sum_{0}^{\infty} \int_{0 \leq t_{1} \leq \cdots \leq t_{n} \leq 1} B\left(t_{1}\right) B\left(t_{2}\right) \ldots B\left(t_{n}\right) \prod d t_{i}
$$


where

$$
B(t)=e^{t A} B e^{-t A} .
$$

One lets $A=-s \log P(0)$ and $B=-s Y$. This gives an equality of the form

$$
\begin{gathered}
P^{-s}=D^{-2 s}-s \int_{0}^{1} \sigma_{-s t}(Y) d t D^{-2 s} \\
+\sum_{2}^{\infty}(-s)^{n} \int_{0 \leq t_{1} \leq \cdots \leq t_{n} \leq 1} \sigma_{-s t_{1}}(Y) \sigma_{-s t_{2}}(Y) \ldots \sigma_{-s t_{n}}(Y) \prod d t_{i}
\end{gathered}
$$

where

$$
\sigma_{u}(T)=\left(D^{2}\right)^{u} T\left(D^{2}\right)^{-u}
$$

One infers from this equality and the absence of poles of order $>1$ in the zeta functions of the form $\operatorname{Tr}\left(Q D^{-2 s}\right)$ that the terms of order $n>1$ in $s$ will not contribute to the value at $s=0$. Thus the following should hold

$$
\zeta_{P}(0)-\zeta_{D^{2}}(0)=-\frac{1}{2} f Y
$$

and using (13) one gets

$$
\zeta_{P}(0)-\zeta_{D^{2}}(0)=f \phi=0,
$$

since the residue vanishes on differential operators. It would take a lot more care to really justify the above manipulations. Instead, in the next section, we shall show by a brute force calculation that $a_{4}$ is independent of $\phi$ so that the above identity is valid.

We thus see that in the first few terms of the spectral action, the only modification we expect when the operator $D^{2}$ is replaced by $P$ is to get an overall factor of $e^{(4-n) \phi}$ multiplying $a_{n}\left(x, D^{2}\right)$ :

$$
\operatorname{Trace}(F(P)) \simeq \sum_{n=0}^{6} f_{n} \int d^{4} x \sqrt{g} e^{(4-n) \phi} a_{n}\left(x, D^{2}\right)+\cdots
$$

Also as will be shown in appendix B, we have the identity

$$
a_{n}\left(x, e^{-\phi} D^{2} e^{-\phi}\right)=a_{n}\left(x, D^{2} e^{-2 \phi}\right)=a_{n}\left(x, e^{-2 \phi} D^{2}\right) .
$$

It is easy to check that by applying the inverse transformation $\phi \rightarrow \phi+\ln m$ one recovers all the $m$ scaling factors obtained in reference [11]. In the next 
section, we shall confirm this result by directly evaluating the spectral action associated with the operator $P$ and in particular the low-energy terms $a_{0}, a_{2}$ and $a_{4}$. We will not attempt to evaluate higher order terms as these are not needed in our analysis.

\section{Dilaton and Seeley-deWitt coefficients}

We compare quite generally the Seeley-deWitt coefficients of an operator $P_{0}=D^{2}$ given by (1) and those of the rescaled operator $P=e^{-\phi} D^{2} e^{-\phi}$. We use the rescaled metric $G$ in the Einstein frame, where the dilaton factor is absorbed in the metric. First we write

$$
P=e^{-\phi} D^{2} e^{-\phi}=-\left(G^{\mu \nu} \partial_{\mu} \partial_{\nu}+\mathcal{A}^{\mu} \partial_{\mu}+\mathcal{B}\right),
$$

where

$$
\begin{aligned}
G^{\mu \nu} & =e^{-2 \phi} g^{\mu \nu}, \\
\mathcal{A}^{\mu} & =e^{-2 \phi} A^{\mu}-2 G^{\mu \nu} \partial_{\nu} \phi, \\
\mathcal{B} & =e^{-2 \phi} B+G^{\mu \nu}\left(\partial_{\mu} \phi \partial_{\nu} \phi-\partial_{\mu} \partial_{\nu} \phi\right)-e^{-2 \phi} A^{\mu} \partial_{\mu} \phi .
\end{aligned}
$$

The Seeley-deWitt coefficients for $a_{n}(P)$ are expressed in terms of $\mathcal{E}$ and $\boldsymbol{\Omega}_{\mu \nu}$ defined by (6) so that,

$$
\begin{aligned}
\mathcal{E} & =\mathcal{B}-G^{\mu \nu}\left(\partial_{\mu}{\overline{\omega^{\prime}}}_{\nu}+{\overline{\omega^{\prime}}}_{\mu}{\overline{\omega^{\prime}}}_{\nu}-\Gamma_{\mu \nu}^{\rho}(G) \overline{\omega^{\prime}}{ }_{\rho}\right), \\
{\overline{\omega^{\prime}}}_{\mu} & =\frac{1}{2} G_{\mu \nu}\left(\mathcal{A}^{\nu}+\Gamma^{\nu}(G)\right), \\
\Omega_{\mu \nu} & =\partial_{\mu}{\overline{\omega^{\prime}}}_{\nu}-\partial_{\nu}{\overline{\omega^{\prime}}}_{\mu}+\left[\overline{\omega^{\prime}}{ }_{\mu},{\overline{\omega^{\prime}}}_{\nu}\right] .
\end{aligned}
$$

These relations imply that

$$
{\overline{\omega^{\prime}}}_{\mu}=\frac{1}{2} g_{\mu \nu} A^{\nu}-\partial_{\mu} \phi+\frac{1}{2} G_{\mu \nu} \Gamma^{\nu}(G) .
$$

The conformal transformations of the Christoffel connection give

$$
\begin{aligned}
\Gamma_{\nu \rho}^{\mu}(G) & =\Gamma_{\nu \rho}^{\mu}(g)+\left(\delta_{\nu}^{\mu} \partial_{\rho} \phi+\delta_{\rho}^{\mu} \partial_{\nu} \phi-g_{\nu \rho} g^{\mu \sigma} \partial_{\sigma} \phi\right), \\
\Gamma^{\mu}(G) & =e^{-2 \phi} \Gamma^{\mu}(g)-2 e^{-2 \phi} g^{\mu \nu} \partial_{\nu} \phi .
\end{aligned}
$$


Using these relations we finally get

$$
\begin{aligned}
{\overline{\omega^{\prime}}}_{\mu} & =\bar{\omega}_{\mu}-2 \partial_{\mu} \phi, \\
\mathcal{E} & =e^{-2 \phi}\left(E+g^{\mu \nu}\left(\nabla_{\mu}^{g} \nabla_{\nu}^{g} \phi+\partial_{\mu} \phi \partial_{\nu} \phi\right)\right),
\end{aligned}
$$

where the covariant derivative $\nabla_{\mu}^{g}$ is taken with respect to the metric $g$. It is quite striking that the perturbation is only a scalar multiple of the identity matrix and does not involve the endomorphisms $A^{\mu}$ at all.

The term $a_{0}$ only involves $\sqrt{G} \operatorname{Tr}(1)$ which, when expressed in terms of the metric $g$ gives $\sqrt{g} e^{4 \phi} \operatorname{Tr}(1)$.

The $a_{2}$ term is proportional to

$$
\int d^{4} x \sqrt{G} \operatorname{Tr}\left(\mathcal{E}-\frac{1}{6} R(G)\right),
$$

where the curvature scalar is constructed as function of the metric $G$. We now use (16) and the relation

$$
R(G)=e^{-2 \phi}\left(R(g)+6 g^{\mu \nu}\left(\nabla_{\mu}^{g} \nabla_{\nu}^{g} \phi+\partial_{\mu} \phi \partial_{\nu} \phi\right)\right)
$$

to obtain at the level of endomorphisms (before taking the fiberwise trace)

$$
\mathcal{E}-\frac{1}{6} R(G)=e^{-2 \phi}\left(E-\frac{1}{6} R(g)\right) \text {. }
$$

This of course implies the required rescaling of the $a_{2}$ term in the required generality, but it is more precise since it holds before taking the trace. We shall use this more precise form in the proof of the invariance of the $a_{4}$ term.

The term $a_{4}(P)$ is given by

$$
\begin{aligned}
\frac{1}{16 \pi^{2}} \frac{1}{360} \int d^{4} x \sqrt{G} \operatorname{Tr} & {\left[\left(5 R^{2}(G)-2 R_{\mu \nu}(G) R^{\mu \nu}(G)+2 R_{\mu \nu \rho \sigma}(G) R^{\mu \nu \rho \sigma}(G)\right)\right.} \\
& \left.-60 R(G) \mathcal{E}+180 \mathcal{E}^{2}+30 \Omega_{\mu \nu} \Omega_{\rho \sigma} G^{\mu \rho} G^{\nu \sigma}\right],
\end{aligned}
$$

where we have omitted the total derivative terms $12(-R(G)+5 \mathcal{E})_{; \mu}{ }^{\mu}$. Since the modification from $\bar{\omega}_{\mu}$ to $\bar{\omega}_{\mu}^{\prime}$ is abelian (15) we get

$$
\Omega_{\mu \nu}=\Omega_{\mu \nu}
$$

and

$$
\boldsymbol{\Omega}_{\mu \nu} \boldsymbol{\Omega}_{\rho \sigma} G^{\mu \rho} G^{\nu \sigma}=e^{-4 \phi} \Omega_{\mu \nu} \Omega_{\rho \sigma} g^{\mu \rho} g^{\nu \sigma} .
$$


Next we group the terms

$$
180 \mathcal{E}^{2}-60 \mathcal{E} R(G)+5 R^{2}(G)=180\left(\mathcal{E}-\frac{1}{6} R(G)\right)^{2}
$$

which yields upon using equation( 18)

$$
180 e^{-4 \phi}\left(E-\frac{1}{6} R(g)\right)^{2}
$$

We are left with the terms

$$
30 \Omega_{\mu \nu} \Omega_{\rho \sigma} G^{\mu \rho} G^{\nu \sigma}-2 R_{\mu \nu}(G) R^{\mu \nu}(G)+2 R_{\mu \nu \rho \sigma}(G) R^{\mu \nu \rho \sigma}(G) .
$$

We now use

$$
\begin{gathered}
30 \operatorname{Tr}\left(\Omega_{\mu \nu} \Omega_{\rho \sigma} G^{\mu \rho} G^{\nu \sigma}\right)=30 e^{-4 \phi} \operatorname{Tr}\left(\Omega_{\mu \nu} \Omega_{\rho \sigma} g^{\mu \rho} g^{\nu \sigma}\right) . \\
-2 R_{\mu \nu}(G) R^{\mu \nu}(G)+2 R_{\mu \nu \rho \sigma}(G) R^{\mu \nu \rho \sigma}(G)=-R(G)^{*} R^{*}(G)+3 C_{\mu \nu \rho \sigma}(G) C^{\mu \nu \rho \sigma}(G) .
\end{gathered}
$$

In deriving the last relation we made use of the two identities

$$
\begin{aligned}
R^{*} R^{*}-R^{2} & =R_{\mu \nu \rho \sigma}^{2}-4 R_{\mu \nu}^{2}, \\
C_{\mu \nu \rho \sigma}^{2}-\frac{1}{3} R^{2} & =R_{\mu \nu \rho \sigma}^{2}-2 R_{\mu \nu}^{2},
\end{aligned}
$$

where $C_{\mu \nu \rho \sigma}$ is the conformal tensor and

$$
R^{*} R^{*}=\frac{1}{4 \sqrt{g}} \epsilon^{\mu \nu \rho \sigma} \epsilon_{\alpha \beta \gamma \delta} R_{\mu \nu}^{\alpha \beta} R_{\rho \sigma}^{\gamma \delta}
$$

These imply

$$
\begin{aligned}
R_{\mu \nu \rho \sigma}^{2} & =2 C_{\mu \nu \rho \sigma}^{2}-R^{*} R^{*}+\frac{1}{3} R^{2} \\
R_{\mu \nu}^{2} & =\frac{1}{2} C_{\mu \nu \rho \sigma}^{2}-\frac{1}{2} R^{*} R^{*}+\frac{1}{3} R^{2} .
\end{aligned}
$$

The square of the conformal tensor is known to be conformal invariant

$$
\int d^{4} x \sqrt{G} C_{\mu \nu \rho \sigma}(G) C^{\mu \nu \rho \sigma}(G)=\int d^{4} x \sqrt{g} C_{\mu \nu \rho \sigma}(g) C^{\mu \nu \rho \sigma}(g) .
$$


The topological Gauss-Bonnet term is metric independent and therefore conformal invariant

$$
\int d^{4} x \sqrt{G} R(G)^{*} R^{*}(G)=\int d^{4} x \sqrt{g} R(g)^{*} R^{*}(g),
$$

and this can be rewritten as

$$
\frac{1}{4} \int d^{4} x \frac{1}{\sqrt{g}} \epsilon^{\mu \nu \rho \sigma} \epsilon_{\alpha \beta \gamma \delta} R_{\mu \nu}^{\alpha \beta} R_{\rho \sigma}^{\gamma \delta} .
$$

This shows that the $a_{4}$ term has the expected invariance under the rescaling of the operator $P_{0} \rightarrow P=e^{-\phi} P_{0} e^{-\phi}$.

\section{Spectral action with dilaton}

We now use the result of the previous section to compute the spectral action with dilaton as a function of the rescaled metric $G$ in the Einstein frame, where the dilaton factor is absorbed in the metric.

The lowest term in the spectral action is given by:

$$
\frac{45}{4 \pi^{2}} f_{0} \int d^{4} x \sqrt{g} e^{4 \phi}=\frac{45}{4 \pi^{2}} f_{0} \int d^{4} x \sqrt{G}
$$

(Note that the dimension of the bundle on which the operator is acting is $4 \times 3 \times 15$ where the 4 is the dimension of spinors, 3 the number of generations and $15=4 \times 3+3$ is the content of each generation).

The next term in the spectral action with dilaton of the standard model is, in terms of the original metric $g$ :

$$
\frac{3}{4 \pi^{2}} f_{2} \int d^{4} x \sqrt{g} e^{2 \phi}\left(\frac{5}{4} R(g)-2 y^{2} H^{*} H\right) .
$$

We can transform this back to the Einstein frame with metric $G_{\mu \nu}$ so that the curvature scalar term has no scale factors in front of it. Using (17) with $g \rightarrow G$ and $\phi \rightarrow-\phi$ the curvature $R(g)$ is

$$
R(g)=e^{2 \phi}\left(R(G)+6 G^{\mu \nu}\left(-\nabla_{\mu}^{G} \nabla_{\nu}^{G} \phi+\partial_{\mu} \phi \partial_{\nu} \phi\right)\right),
$$


and we obtain

$$
\begin{aligned}
\int d^{4} x \sqrt{g} e^{2 \phi} R(g) & =\int d^{4} x \sqrt{G}\left(R(G)+6 G^{\mu \nu}\left(-\nabla_{\mu}^{G} \nabla_{\nu}^{G} \phi+\partial_{\mu} \phi \partial_{\nu} \phi\right)\right) \\
& =\int d^{4} x \sqrt{G}\left(R(G)+6 G^{\mu \nu} \partial_{\mu} \phi \partial_{\nu} \phi\right)
\end{aligned}
$$

after integrating by parts. The $a_{2}$ term (19) thus becomes

$$
\frac{3}{4 \pi^{2}} f_{2} \int d^{4} x \sqrt{G}\left(\frac{5}{4} R(G)+\frac{15}{2} G^{\mu \nu} \partial_{\mu} \phi \partial_{\nu} \phi-2 y^{2} H^{\prime *} H^{\prime}\right)
$$

where we have defined

$$
H=e^{\phi} H^{\prime},
$$

so that the only appearance of the dilaton $\phi$ is through its kinetic energy.

Let us pause a bit and discuss signs at this point. For a positive test function $F$ the coefficients $f_{0}, f_{2}, f_{4}$ are all positive. It is important that the Einstein term $\int d^{4} x \sqrt{G} R(G)$ appears in (20) with the correct sign for the Euclidean functional integral, and that the kinetic term for $\phi$ namely $\int d^{4} x \sqrt{G} G^{\mu \nu} \partial_{\mu} \phi \partial_{\nu} \phi$ appears with a positive coefficient in (20).

The next term coming from $a_{4}(x, P)$ is unchanged for the spectral action with dilaton, and thus given independently of $\phi$ by [11],

$$
\begin{aligned}
\frac{f_{4}}{4 \pi^{2}} \int d^{4} x \sqrt{g} & \left(\frac{1}{32}\left(11 R(g)^{*} R^{*}(g)-18 C_{\mu \nu \rho \sigma}(g) C_{\alpha \beta \gamma \delta}(g) g^{\mu \alpha} g^{\nu \beta} g^{\rho \gamma} g^{\sigma \delta}\right)\right. \\
& +3 y^{2}\left(D_{\mu} H^{*} D_{\nu} H g^{\mu \nu}-\frac{1}{6} R(g) H^{*} H\right) \\
& \left.+\left(g_{3}^{2} G_{\mu \nu}^{i} G_{\rho \sigma}^{i}+g_{2}^{2} F_{\mu \nu}^{\alpha} F_{\rho \sigma}^{\alpha}+\frac{5}{3} g_{1}^{2} B_{\mu \nu} B_{\rho \sigma}\right) g^{\mu \rho} g^{\nu \sigma}+3 z^{2}\left(H^{*} H\right)^{2}\right),
\end{aligned}
$$

where we have omitted total derivatives as they only contribute to boundary terms. Let us show that we can rewrite this term in the following way as a function of the metric $G_{\mu \nu}$ by making use of the conformal invariance of $a_{4}$ :

$$
\begin{aligned}
\frac{f_{4}}{4 \pi^{2}} \int d^{4} x \sqrt{G} & \left(\frac{1}{32}\left(11 R(G)^{*} R^{*}(G)-18 C_{\mu \nu \rho \sigma}(G) C_{\alpha \beta \gamma \delta}(G) G^{\mu \alpha} G^{\nu \beta} G^{\rho \gamma} G^{\sigma \delta}\right)\right. \\
& +3 y^{2}\left(D_{\mu} H^{\prime *} D_{\nu} H^{\prime} G^{\mu \nu}-\frac{1}{6} R(G) H^{\prime *} H^{\prime}\right) \\
& \left.+\left(g_{3}^{2} G_{\mu \nu}^{i} G_{\rho \sigma}^{i}+g_{2}^{2} F_{\mu \nu}^{\alpha} F_{\rho \sigma}^{\alpha}+\frac{5}{3} g_{1}^{2} B_{\mu \nu} B_{\rho \sigma}\right) G^{\mu \rho} G^{\nu \sigma}+3 z^{2}\left(H^{\prime *} H^{\prime}\right)^{2}\right) .
\end{aligned}
$$


The terms which only involve the metric are conformal by construction. The same holds for the terms which involve the gauge fields since the YangMills action is conformal. Thus we need only to take care of the terms that involve the Higgs fields. We have to show that the following expression is unchanged by $g \rightarrow G$ and $H \rightarrow H^{\prime}$,

$$
\frac{3 f_{4} y^{2}}{4 \pi^{2}} \int d^{4} x \sqrt{g}\left(g^{\mu \nu} D_{\mu} H^{*} D_{\nu} H-\frac{1}{6} R(g) H^{*} H\right) .
$$

To see this we first rescale the kinetic energy of the Higgs field

$$
\begin{aligned}
\sqrt{g} g^{\mu \nu} D_{\mu} H^{*} D_{\nu} H & =\sqrt{G} G^{\mu \nu} e^{-2 \phi} D_{\mu}\left(e^{\phi} H^{\prime *}\right) D_{\nu}\left(e^{\phi} H^{\prime}\right) \\
& =\sqrt{G} G^{\mu \nu}\left(D_{\mu} H^{\prime *} D_{\nu} H^{\prime}+\partial_{\mu} \phi H^{\prime *} D_{\nu} H^{\prime}\right. \\
& \left.+D_{\mu} H^{\prime *} H^{\prime} \partial_{\nu} \phi+H^{\prime *} H^{\prime} \partial_{\mu} \phi \partial_{\nu} \phi\right) .
\end{aligned}
$$

The conformal coupling of the Higgs field to the scalar curvature transforms as

$$
-\frac{1}{6} \sqrt{g} R(g) H^{*} H=-\frac{1}{6} \sqrt{G} H^{\prime *} H^{\prime}\left(R(G)-6 G^{\mu \nu}\left(\left(\nabla_{\mu} \nabla_{\nu}\right)^{G} \phi-\partial_{\mu} \phi \partial_{\nu} \phi\right)\right) .
$$

After integrating by parts the term

$$
\int d^{4} x \sqrt{G} H^{*} H^{\prime}\left(\nabla_{\mu} \nabla_{\nu}\right)^{G} \phi G^{\mu \nu},
$$

we find that all cross terms cancel, thus obtaining

$$
\begin{aligned}
& \int d^{4} x \sqrt{g}\left(g^{\mu \nu} D_{\mu} H^{*} D_{\nu} H-\frac{1}{6} R(g) H^{*} H\right) \\
& =\int d^{4} x \sqrt{G}\left(G^{\mu \nu} D_{\mu} H^{\prime *} D_{\nu} H^{\prime}-\frac{1}{6} R(G) H^{\prime *} H^{\prime}\right) .
\end{aligned}
$$

The quartic Higgs interactions are evidently scale invariant

$$
\int d^{4} x \sqrt{g}\left(H^{*} H\right)^{2}=\int d^{4} x \sqrt{G}\left(H^{* *} H^{\prime}\right)^{2} .
$$

Collecting all terms, the low-energy bosonic part of the spectral action with 
dilaton is given by

$$
\begin{aligned}
& I_{b}=\frac{45}{4 \pi^{2}} f_{0} \int d^{4} x \sqrt{G} \\
& +\frac{3}{4 \pi^{2}} f_{2} \int d^{4} x \sqrt{G}\left(\frac{5}{4} R(G)+\frac{15}{2} G^{\mu \nu} \partial_{\mu} \phi \partial_{\nu} \phi-2 y^{2} H^{\prime *} H^{\prime}\right) \\
& +\frac{f_{4}}{4 \pi^{2}} \int d^{4} x \sqrt{G}\left(\frac{1}{32}\left(11 R(G)^{*} R^{*}(G)-18 C_{\mu \nu \rho \sigma}(G) C^{\mu \nu \rho \sigma}(G)\right)\right. \\
& \quad+3 y^{2}\left(D_{\mu} H^{\prime *} D_{\nu} H^{\prime} G^{\mu \nu}-\frac{1}{6} R(G) H^{\prime *} H^{\prime}\right) \\
& \left.\quad+\left(g_{3}^{2} G_{\mu \nu}^{i} G_{\rho \sigma}^{i}+g_{2}^{2} F_{\mu \nu}^{\alpha} F_{\rho \sigma}^{\alpha}+\frac{5}{3} g_{1}^{2} B_{\mu \nu} B_{\rho \sigma}\right) G^{\mu \rho} G^{\nu \sigma}+3 z^{2}\left(H^{\prime *} H^{\prime}\right)^{2}\right) .
\end{aligned}
$$

For higher order terms one expects a scaling factor of the form $e^{(4-n) \phi}$ to be present, but derivatives of the dilaton field $\phi$ may also occur. Therefore in the Einstein frame, one does no expect the dilaton field $\phi$ to acquire a potential. As will be discussed later, this will change when quantum corrections are taken into account and the dilaton acquires a potential of the Coleman-Weinberg type [14].

Fermionic interactions take the simple form

$$
\langle\Psi|D| \Psi\rangle=\int d^{4} x \sqrt{g} \bar{\Psi} D \Psi
$$

where the metric $g_{\mu \nu}$ is used to insure hermiticity of $D$. We will now show that the fermions will not feel the dilaton. To see this we first redefine the spinors by

$$
\Psi=e^{\frac{3}{2} \phi} \Psi^{\prime}
$$

then we have, for the parts not involving the Higgs or gauge fields,

$$
\langle\Psi|D| \Psi\rangle=\int d^{4} x \sqrt{G} e^{-4 \phi} e^{\frac{3}{2} \phi} \bar{\Psi}^{\prime} \gamma^{c} e^{\phi} E_{c}^{\mu}\left(\partial_{\mu}+\frac{1}{4} \omega_{\mu}^{a b}(e) \gamma_{a b}\right)\left(e^{\frac{3}{2} \phi} \Psi^{\prime}\right),
$$

where the rescaled vierbein is $E_{\mu}^{a}=e^{\phi} e_{\mu}^{a}$. We have to express the spinconnection $\omega_{\mu}^{a b}(e)$ in terms of the spin-connection of the rescaled vierbein $\Omega_{\mu}^{a b}(E)$. To do this we use the equations

$$
\begin{aligned}
\partial_{\mu} e_{\nu}^{a}-\partial_{\nu} e_{\mu}^{a}-\omega_{\mu}^{a b}(e) e_{\nu b}+\omega_{\nu}^{a b}(e) e_{\mu b} & =0 \\
\partial_{\mu} E_{\nu}^{a}-\partial_{\nu} E_{\mu}^{a}-\Omega_{\mu}^{a b}(E) E_{\nu b}+\Omega_{\nu}^{a b}(E) E_{\mu b} & =0
\end{aligned}
$$


and these imply that

$$
\omega_{\mu}^{a b}(e)=\Omega_{\mu}^{a b}(E)+\left(e_{\mu}^{a} e^{\nu b}-e_{\mu}^{a} e^{\nu b}\right) \partial_{\nu} \phi .
$$

Therefore

$$
\gamma^{c} e_{c}^{\mu}\left(\frac{3}{2} \partial_{\mu} \phi+\frac{1}{4} \omega_{\mu}^{a b}(e) \gamma_{a b}\right)=\gamma^{c} e^{\phi} E_{c}^{\mu}\left(\frac{1}{4} \Omega_{\mu}^{a b}(E) \gamma_{a b}\right),
$$

and the fermionic action reduces to the nice form

$$
\int d^{4} x \sqrt{G} \bar{\Psi}^{\prime} \gamma^{c} E_{c}^{\mu}\left(\partial_{\mu}+\frac{1}{4} \Omega_{\mu}^{a b}(E) \gamma_{a b}\right) \Psi^{\prime}
$$

which is independent of the dilaton. Finally the parts involving interactions between the fermions and the Higgs or gauge fields could be written in the form

$$
\begin{gathered}
\int d^{4} x \sqrt{g} \bar{\Psi} \gamma_{5} H \Psi=\int d^{4} x \sqrt{G} \overline{\Psi^{\prime}} \gamma_{5} H^{\prime} \Psi^{\prime} \\
\int d^{4} x \sqrt{g} \bar{\Psi} \gamma^{a} e_{a}^{\mu} A_{\mu} \Psi=\int d^{4} x \sqrt{G} \overline{\Psi^{\prime}} \gamma^{a} E_{a}^{\mu} A_{\mu} \Psi^{\prime}
\end{gathered}
$$

The fermionic interactions are

$$
I_{f}=\left\langle Q\left|D_{q}\right| Q\right\rangle+\left\langle L\left|D_{l}\right| L\right\rangle
$$

where

$$
Q=\left(\begin{array}{c}
u_{L} \\
d_{L} \\
d_{R} \\
u_{R}
\end{array}\right), \quad\left(\begin{array}{c}
\nu_{L} \\
e_{L} \\
e_{R}
\end{array}\right)
$$

and these take exactly the same form as those without dilaton when expressed in terms of the metric $G_{\mu \nu}$. Rewriting this in terms of the fermionic fields

$$
Q^{\prime}=e^{-\frac{3}{2} \phi} Q, \quad L^{\prime}=e^{-\frac{3}{2} \phi} L,
$$

and the Higgs field $H^{\prime}$ we obtain

$$
I_{f}=\int d^{4} x \sqrt{G}\left(\bar{L}^{\prime} D_{l}^{\prime} L^{\prime}+\bar{Q}^{\prime} D_{q}^{\prime} Q^{\prime}\right)
$$


where [11]

$$
\begin{aligned}
& D_{l}^{\prime}=\left(\begin{array}{cc}
\gamma^{\mu} \otimes\left(D_{\mu} \otimes 1_{2}-\frac{i}{2} g_{2} A_{\mu}^{\alpha} \sigma^{\alpha}+\frac{i}{2} g_{1} B_{\mu} \otimes 1_{2}\right) \otimes 1_{3} & \gamma_{5} \otimes k^{e} \otimes H^{\prime} \\
\gamma_{5} \otimes k^{* e} \otimes H^{*^{\prime}} & \gamma^{\mu} \otimes\left(D_{\mu}+i g_{1} B_{\mu}\right) \otimes 1_{3}
\end{array}\right) \\
& D_{q}^{\prime}=\left(\begin{array}{ccc}
\gamma^{\mu} \otimes \nabla_{\mu}^{(1,2)} \otimes 1_{3} & \gamma_{5} \otimes k^{d} \otimes H^{\prime} & \gamma_{5} \otimes k^{u} \otimes \widetilde{H}^{\prime} \\
\gamma_{5} \otimes k^{* d} \otimes H^{*^{\prime}} & \gamma^{\mu} \otimes\left(D_{\mu}+\frac{i}{3} g_{1} B_{\mu}\right) \otimes 1_{3} & 0 \\
\gamma_{5} \otimes k^{* u} \otimes \widetilde{H}^{\prime *} & 0 & \gamma^{\mu} \otimes\left(D_{\mu}-\frac{2 i}{3} g_{1} B_{\mu}\right) \otimes 1_{3}
\end{array}\right) \\
& +\gamma^{\mu} \otimes 1_{4} \otimes 1_{3} \otimes\left(-\frac{i}{2} g_{3} V_{\mu}^{i} \lambda^{i}\right)
\end{aligned}
$$

and

$$
\begin{aligned}
\gamma^{\mu} & =\gamma^{a} E_{a}^{\mu}, \\
D_{\mu} & =\partial_{\mu}+\frac{1}{4} \Omega_{\mu}^{a b}(E) \gamma_{a b}, \\
\nabla_{\mu}^{(1,2)} & =D_{\mu} \otimes 1_{2}-\frac{i}{2} g_{2} A_{\mu}^{\alpha} \sigma^{\alpha}-\frac{i}{6} g_{1} B_{\mu} \otimes 1_{2} .
\end{aligned}
$$

From the above considerations we deduce that the only effect of the dilaton on the low-energy terms of the spectral action is that the dilaton gets a kinetic term with no other interactions. This confirms that all matter interactions in the above Lagrangian are scale invariant when expressed in the rescaled fields $G_{\mu \nu}, H^{\prime}$ and $\Psi^{\prime}$. Only the Einstein term and the dilaton kinetic energy are not scale invariant.

Note that the invariance of the action for the Fermions, that is the equality

$$
\langle\Psi|D| \Psi\rangle=\left\langle\Psi^{\prime}\left|D^{\prime}\right| \Psi^{\prime}\right\rangle^{\prime}
$$

where $D^{\prime}$ corresponds to the metric $G$ and the fields $H^{\prime}$, does not mean that the operators $D$ and $D^{\prime}$ are the same. Indeed the transformation $\Psi \rightarrow \Psi^{\prime}$ is not unitary and one has

$$
\left\langle\Psi^{\prime} \mid \Psi^{\prime}\right\rangle^{\prime}=\left\langle\Psi\left|e^{\phi}\right| \Psi\right\rangle
$$

which gives the unitary equivalence

$$
D^{\prime} \sim e^{-\phi / 2} D e^{-\phi / 2}
$$


One might then be tempted to conclude that the square of $e^{-\phi / 2} D e^{-\phi / 2}$ should be unitarily equivalent to $P=e^{-\phi} D^{2} e^{-\phi}$ but this does not hold precisely because of the additional kinetic term in the spectral action with dilaton. Indeed one can prove (Appendix C) in the general framework of spectral triples, with a minimum amount of hypothesis, the identity

$$
f e^{2 \phi} D^{-2}=f\left(e^{-\phi / 2} D e^{-\phi / 2}\right)^{-2}+\frac{1}{2} f\left[D, e^{\phi}\right]\left[D, e^{\phi}\right]^{*} D^{-4}
$$

with $D^{\prime}=e^{-\phi / 2} D e^{-\phi / 2}$ the last term gives the canonical kinetic energy of the dilaton

$$
\frac{1}{2} f\left[D^{\prime}, \phi\right]\left[D^{\prime}, \phi\right]^{*} D^{\prime-4}
$$

with the correct sign.

\section{Applications}

We have shown that the dilaton interactions of the spectral action are almost the same as the ones proposed in the literature [2], [3], the difference lies in the derivative couplings of the dilaton field. These were obtained by requiring the standard model matter sector to be scale invariant by introducing a compensating dilaton field. The origin of the dilatational symmetry breaking are the mass terms of the Higgs potential, and these are scaled with the dilaton field to make them scale invariant. In a curved space-time all fields couple to gravity, and the dilaton. The proposed action for the gravitydilaton-Higgs sectors, in our notation, was derived to be ${ }^{2}[3],[4]$

$$
\begin{aligned}
I=\int d^{4} x \sqrt{G} & \left(-\frac{1}{2 \kappa^{2}} R+\frac{1}{2}\left(1+\frac{6}{\kappa^{2} f^{2}}\right) G^{\mu \nu} \partial_{\mu} \phi \partial_{\nu} \phi\right. \\
& \left.+G^{\mu \nu} D_{\mu} H^{*} D_{\nu} H^{\prime}-V_{0}\left(H^{*} H^{\prime}\right) .\right)
\end{aligned}
$$

There it was shown that in curved space-time this corresponds to the JordanBrans-Dicke theory of gravity. The only difference between this action and the spectral action is that the later has the conformal coupling

$$
\frac{1}{6} R(G)\left(H^{*} H^{\prime}\right)^{2}
$$

\footnotetext{
${ }^{2}$ This expression is in the conventions of [4] and is in Minkowski space.
} 
which is necessary to make the matter couplings scale invariant. A slight modification was also proposed in the study of models of extended inflation, by also considering the possibility of modifying the Higgs sector by taking $[4]$

$$
e^{\frac{2}{f} \phi} G^{\mu \nu} D_{\mu} H^{*} D_{\nu} H-e^{\frac{4}{f} \phi} V_{0}\left(H^{*} H\right)
$$

This differs from the spectral action by the appearance of derivative couplings of the form

$$
G^{\mu \nu} D_{\mu} H^{*} H^{\prime} \partial_{\nu} \phi
$$

It is amusing to note that this alternative proposed action is exactly the same action as the one derived for the Connes-Lott gravitational interactions [6], [10]. Therefore the two models proposed in the literature for making the Higgs sector scale invariant are the same as the interactions obtained for the noncommutative standard model, either for the spectral action formulation, or the Connes-Lott formulation. We also note that scale invariance of the action is broken by the Einstein term and by the kinetic term for the dilaton. This is remarkable because it was shown that if the full action is scale invariant, then the couplings will not lead to a model with extended inflation. Quantum corrections and renormalization conditions break scale invariance in the matter sector of the standard model and lead to an exponentially large hierarchy between the mass scale $f$ where $\phi=\frac{1}{f} \sigma$ and the electroweak scale without fine tuning. The scale $f$ is normally of the order of the Planck scale. The dilaton mass obtained depends on the Higgs mass, but should be constrained to be smaller than $10^{-6} \mathrm{ev}$.

The noncommutative space of the standard model is obtained by taking the product of a four-dimensional Riemannian manifold times a discrete space dictated by the symmetries of the Hilbert space spanned by the quarks and leptons. The presence of left and right handed fermions provides the intuitive picture where these fermions are placed on two different sheets. The gauge fields in the discrete dimensions are the Higgs fields, with the inverse of the distance between the two sheets interpreted as the electroweak energy scale. This picture is similar to the Randall-Sundrum (RS) scenario where the fourdimensional space is embedded into a five-dimensional space as a 3-brane positioned at the points $x_{5}=0$ and $x_{5}=\pi r_{c}$ where $r_{c}$ is the compactification radius. The action for the Higgs sector in the RS model [9] was obtained to 
be

$$
\begin{aligned}
& \int d^{4} x \sqrt{g}\left(g^{\mu \nu} D_{\mu} H^{*} D_{\nu} H-\lambda\left(|H|^{2}-v_{0}^{2}\right)\right) \\
= & \int d^{4} x \sqrt{\bar{g}}\left(\bar{g}^{\mu \nu} D_{\mu} H^{\prime *} D_{\nu} H^{\prime}-\lambda\left(\left|H^{\prime}\right|^{2}-e^{-2 k r_{c} \pi} v_{0}^{2}\right)\right)
\end{aligned}
$$

where

$$
\begin{aligned}
g^{\mu \nu} & =e^{-2 k r_{c} \pi} \bar{g}^{\mu \nu}, \\
H^{\prime} & =e^{2 k r_{c} \phi} H,
\end{aligned}
$$

in the visible sector located at $x_{5}=\pi r_{c}$. The physical mass scales are set by the symmetry breaking scale $v=e^{-k r_{c} \pi} v_{0}$ so that $m=m_{0} e^{-k r_{c} \pi}$. The bare symmetry breaking scale $v_{0}$ is taken to be of the order of the Planck scale at $10^{19} \mathrm{Gev}$ and the scaling factor $e^{k r_{c} \pi}$ tuned to be of the order of $10^{15} \mathrm{so}$ that the low-energy masses are of the order of Tev. The hierarchy problem is only partially solved in a technical sense because the tuning could not be maintained at the quantum level. A choice of $k r_{c}=10$ can generate the large scale $10^{15}$ Gev. Comparing the Higgs sectors in the RS action with that in the spectral action we immediately see that they are identical provided we identify the expectation value of the dilaton field $\langle\phi\rangle$ with $k r_{c} \pi$.

\section{Conclusions}

The Dirac operator being a differential operator has the dimensions of mass. The spectral action in noncommutative geometry is defined as a function of a dimensionless operator which is taken to be the Dirac operator divided by some arbitrary large mass scale. The arbitrariness of the mass scale naturally suggests to make this scale dynamical by introducing a dilaton field in the Dirac operator of the noncommutative space defined by the standard model. To understand the appearance of the mass scales of the spectral action, we evaluated all interactions of the dilaton with the matter sector in the standard model. We found the remarkable result that the low-energy action, when evaluated in the Einstein frame, is scale invariant except for the Einstein-Hilbert term and the dilaton kinetic term. The resulting model is almost identical to the one proposed in the literature [3],[2],[4]. The main motivation in these works is the observation that the standard model is classically almost scale invariant, with the symmetry only broken by the mass term 
in the Higgs potential. The symmetry is restored by the use of a dilaton field. When coupled to gravity, neither the dilaton kinetic energy nor the scalar curvature are scale invariant, leading to a Jordan-Brans-Dicke theory of gravity. The vacuum expectation value of the Higgs field is then dependent on the dilaton and is classically undetermined. Quantum corrections break the scale invariance of the scalar potential and change the vacuum expectation value of the Higgs field. The dilaton acquires a large negative expectation value given by $-m$ and a small mass. The hierarchy in mass scales is due to the large Yukawa coupling of the top quark. The dilaton expectation value can range between the GUT scale of $10^{15} \mathrm{Gev}$ to the Planck scale of $2.4 \cdot 10^{18}$ Gev. The hierarchy in mass scales is not possible if the dilaton kinetic energy and the gravitational action were scale invariant. It is remarkable that all the essential features of building a scale invariant standard model interactions to generate a mass hierarchy and predict the Higgs mass are naturally included in the spectral action without any fine tuning. It is worth mentioning that the scalar potential of exactly the same model considered here was shown to admit extended inflation and a metastable ground state. It also evades the problems of the original version of extended inflation.

The vacuum expectation value of the dilaton field is determined by getting contributions from classical and radiative corrections to the vacuum energy density. One does not obtain naturally a vanishing cosmological constant. There are two possibilities to cure this problem. The first is to determine the low-energy value of the cosmological constant as determined by the renormaliztion group equations and then fine tune this value to cancel the contributions of the Coleman-Weinberg potential. The second possibility to cure this problem is to fix the total invariant volume. This restricts general relativity to the form considered in [15] where the volume form is held fixed. There it was shown that this picture is consistent both at the classical and quantum levels [16], [17],[18] . This fixes the total invariant volume and eliminates the scalar mode of the metric tensor $g_{\mu \nu}$. This is done at the expense of introducing the dilaton mode $\phi$ [3]. In noncommutative geometry the volume is fixed by a Hochschild cycle $c$ whose compatibility with the Dirac operator $D$ is a basic constraint on the Hilbert space representation giving the metric [19]. One applies the representation to monomials

$$
\pi\left(f_{0}, f_{1}, f_{2}, f_{3}, f_{4}\right)=f_{0}\left[D, f_{1}\right]\left[D, f_{2}\right]\left[D, f_{3}\right]\left[D, f_{4}\right]
$$


and requires that when applied to the Hochschild cycle $c$ it gives

$$
\pi(c)=\gamma_{5}
$$

The cosmological constant becomes determined by the initial conditions of the theory.

To summarize, we have shown that the spectral action includes naturally a dilaton field which guarantees the scale invariance of the standard model interactions, and provides a mechanism to generate mass hierarchies. This is in addition to the advantages obtained previously in [11] which are now well known [12]. There it was shown that all the correct features of the standard model are obtained without any fine tuning, such as unification with gravity, unification of the three gauge coupling constants and relating the Higgs to the gauge couplings. These results should be taken to support the idea that all the geometric information about the physical space is captured by the knowledge of the Dirac operator of an appropriate noncommutative space.

\section{Appendix A}

In this appendix we shall prove formula (12). Given an elliptic positive invertible second order operator $Q$ and a differential operator $T$ we use the notation $\nabla(T)=[Q, T]$ and the following identity (for $n \geq 0$ ) :

$$
Q^{-1} T=\sum_{0}^{n}(-1)^{k} \nabla^{k}(T) Q^{-k-1}+(-1)^{n+1} Q^{-1} \nabla^{n+1}(T) Q^{-n-1} .
$$

We apply this to $Q=P(t)+\lambda, T=\phi P(t)+P(t) \phi$. The operator $\nabla^{k}(T)$ is independent of $\lambda$ and is a differential operator of order $\leq 2+k$ since $\nabla^{k}(\phi)$ is at most of order $k$. Thus the operator $\nabla^{k}(T) Q^{-k-1}$ is pseudodifferential of order at most $2+k-2(k+1)=-k$ and the remainder in (26) is of order at most $-n-1$. This shows that when working modulo operators of order less than $-n$ we have

$$
(P(t)+\lambda)^{-1} T \sim T(P(t)+\lambda)^{-1}+\sum_{1}^{n}(-1)^{k} \nabla^{k}(T)(P(t)+\lambda)^{-k-1}
$$

so that

$$
(P(t)+\lambda)^{-1} T(P(t)+\lambda)^{-1} \sim T(P(t)+\lambda)^{-2}
$$




$$
+\sum_{1}^{n}(-1)^{k} \nabla^{k}(T)(P(t)+\lambda)^{-k-2}
$$

But one has

$$
\sum_{1}^{n}(-1)^{k} \nabla^{k}(T)(P(t)+\lambda)^{-k-2}=[P(t), A(t, \lambda)]
$$

where

$$
A(t, \lambda)=\sum_{1}^{n}(-1)^{k} \nabla^{k-1}(T)(P(t)+\lambda)^{-k-2}
$$

Thus integrating from $\lambda=0$ to $\infty$ and using (11) we get that, modulo operators of order less than $-n$,

$$
\frac{d}{d t} Y(t) \sim-\int_{0}^{\infty}(\phi P(t)+P(t) \phi)(P(t)+\lambda)^{-2} d \lambda+[P(t), A(t)]
$$

where

$$
A(t)=-\int_{0}^{\infty} A(t, \lambda) d \lambda
$$

This thus gives

$$
\begin{aligned}
& \frac{d}{d t} Y(t) \sim-(\phi P(t)+P(t) \phi) P(t)^{-1}+[P(t), A(t)] \\
& =-2 \phi+[P(t), C(t)], \quad C(t)=A(t)-\phi P(t)^{-1}
\end{aligned}
$$

It is important to note that all of the above manipulations hold in the general context of spectral triples with simple dimension spectrum. Moreover one can prove fairly strong properties of the spectral action in this general context.

\section{Appendix B}

In this appendix we shall prove the identity

$$
a_{n}(x, P)=a_{n}\left(x, P_{1}\right)=a_{n}\left(x, P_{2}\right)
$$


where $P=e^{-\phi} D^{2} e^{-\phi}$ and $P_{1}=D^{2} e^{-2 \phi}, P_{2}=e^{-2 \phi} D^{2}$. It can then be used to simplify some of the computations of section 3 .

One simply writes

$$
P=e^{-\phi} P_{1} e^{\phi}
$$

so that

$$
\text { Trace }\left(P^{-s}\right)=\text { Trace }\left(P_{1}^{-s}\right)
$$

From this the identity $a_{n}(x, P)=a_{n}\left(x, P_{1}\right)$ immediately follows.

One can also do a direct check as follows, one first writes

$$
\begin{gathered}
P=-\left(G^{\mu \nu} I \partial_{\mu} \partial_{\nu}+\mathcal{A}^{\mu} \partial_{\mu}+\mathcal{B}\right) \\
P_{1}=-\left(G^{\mu \nu} I \partial_{\mu} \partial_{\nu}+\mathcal{A}_{1}^{\mu} \partial_{\mu}+\mathcal{B}_{1}\right),
\end{gathered}
$$

where

$$
\begin{aligned}
G^{\mu \nu} & =e^{-2 \phi} g^{\mu \nu} \\
\mathcal{B} & =e^{-2 \phi} B+G^{\mu \nu}\left(\partial_{\mu} \phi \partial_{\nu} \phi-\partial_{\mu} \partial_{\nu} \phi\right)-e^{-2 \phi} A^{\mu} \partial_{\mu} \phi \\
\mathcal{A}^{\mu} & =e^{-2 \phi} A^{\mu}-2 G^{\mu \nu} \partial_{\nu} \phi \\
\mathcal{A}_{1}^{\mu} & =e^{-2 \phi} A^{\mu}-4 G^{\mu \nu} \partial_{\nu} \phi \\
\mathcal{B}_{1} & =e^{-2 \phi} B+2 G^{\mu \nu}\left(2 \partial_{\mu} \phi \partial_{\nu} \phi-\partial_{\mu} \partial_{\nu} \phi\right)-2 e^{-2 \phi} A^{\mu} \partial_{\mu} \phi
\end{aligned}
$$

These relations imply

$$
\begin{aligned}
\mathcal{A}_{1}^{\mu} & =\mathcal{A}^{\mu}-2 G^{\mu \nu} \partial_{\nu} \phi \\
\mathcal{B}_{1} & =\mathcal{B}+G^{\mu \nu}\left(\partial_{\mu} \phi \partial_{\nu} \phi-\partial_{\mu} \partial_{\nu} \phi\right)-\mathcal{A}^{\mu} \partial_{\mu} \phi
\end{aligned}
$$

We also have

$$
\begin{aligned}
{\overline{\omega^{\prime}}}_{1 \mu} & =\frac{1}{2} G_{\mu \nu}\left(\mathcal{A}_{1}^{\nu}+\Gamma^{\nu}(G)\right) \\
& ={\overline{\omega^{\prime}}}_{\mu}-\partial_{\mu} \phi
\end{aligned}
$$

so that

$$
\begin{aligned}
\mathcal{E}_{1} & =\mathcal{B}_{1}-G^{\mu \nu}\left(\partial_{\mu}{\overline{\omega^{\prime}}}_{1 \nu}+{\overline{\omega^{\prime}}}_{1 \mu}{\overline{\omega^{\prime}}}_{1 \nu}-\Gamma_{\mu \nu}^{\rho}(G){\overline{\omega^{\prime}}}_{1 \rho}\right) \\
& =\mathcal{E}
\end{aligned}
$$

Similarly

$$
\Omega_{1 \mu \nu}=\Omega_{\mu \nu}
$$

and the equality of the Seely-de Witt coefficients follow from the fact that these depend only on $\mathcal{E}, \Omega_{\mu \nu}$ and the curvature tensors are functions of the same metric $G_{\mu \nu}$. 


\section{Appendix C}

In this appendix we shall show (25) and the appearance of the kinetic term in the general framework of spectral triples using the following manipulations. One has

$$
f\left(e^{-\phi / 2} D e^{-\phi / 2}\right)^{-2}=f e^{\phi} D^{-1} e^{\phi} D^{-1}
$$

Also

$$
D^{-1} e^{\phi}=e^{\phi} D^{-1}-D^{-1}\left[D, e^{\phi}\right] D^{-1}
$$

which allows to write $(27)$ as

$$
f\left(e^{-\phi / 2} D e^{-\phi / 2}\right)^{-2}=f e^{2 \phi} D^{-2}-f e^{\phi} D^{-1}\left[D, e^{\phi}\right] D^{-2},
$$

and using (28) again,

$-f e^{\phi} D^{-1}\left[D, e^{\phi}\right] D^{-2}=-f e^{\phi}\left[D, e^{\phi}\right] D^{-3}-f D^{-1}\left[D, e^{\phi}\right] D^{-1}\left[D, e^{\phi}\right] D^{-2}$.

The first of the two terms vanishes since the residue is a trace. The second is given by

$$
-f D^{-1}\left[D, e^{\phi}\right] D^{-1}\left[D, e^{\phi}\right] D^{-2}=f\left[D, e^{\phi}\right]^{2} D^{-4}+R,
$$

where $R$ is the remainder

$$
R=-f D^{-2}\left[D^{2}, e^{\phi}\right] D^{-1}\left[D, e^{\phi}\right] D^{-2}
$$

Let us show that

$$
R=-\frac{1}{2} f\left[D^{2}, e^{\phi}\right]^{2} D^{-6}
$$

To see this write

$$
R=-f D^{-2}\left[D^{2}, e^{\phi}\right] D D^{-2}\left[D, e^{\phi}\right] D^{-2}
$$

and note that the commutator of $D$ with $\left[D^{2}, e^{\phi}\right]$ is equal to $\left[D^{2},\left[D, e^{\phi}\right]\right]$ and has order 1 so that

$$
f D^{-2}\left[D,\left[D^{2}, e^{\phi}\right]\right] D^{-2}\left[D, e^{\phi}\right] D^{-2}=0 .
$$


Thus moving $D$ to the left and using the trace property of the residue one gets

$$
R=-\frac{1}{2} f\left[D^{2}, e^{\phi}\right] D^{-2}\left(D\left[D, e^{\phi}\right]+\left[D, e^{\phi}\right] D\right) D^{-4},
$$

and one obtains (31). Summarizing, we have shown the equality

$$
f\left(e^{-\phi / 2} D e^{-\phi / 2}\right)^{-2}=f e^{2 \phi} D^{-2}+f\left[D, e^{\phi}\right]^{2} D^{-4}-\frac{1}{2} f\left[D^{2}, e^{\phi}\right]^{2} D^{-6} .
$$

Thus to obtain (27) one just needs to prove the equality

$$
f[D, a]^{2} D^{-4}=f\left[D^{2}, a\right]^{2} D^{-6},
$$

and apply it to $a=e^{\phi}$. One can check (33) directly in the Riemannian case by computing the residue as the integral of the principal symbols on the unit sphere bundle. The factor $2^{2}$ from the Poisson brackets $\left[D^{2}, a\right]$ is compensated by the integral of $\xi_{\mu}^{2}$ on the sphere which gives $\frac{1}{4}$. In the general framework of spectral triples one gets (33) from the general hypothesis

$$
f a[D, b] D^{-3}=0, \quad \forall a, b \in \mathcal{A} .
$$

Note that the commutator $\left[D, e^{\phi}\right]$ is skew adjoint and in particular $\left[D, e^{\phi}\right]^{*}=$ $-\left[D, e^{\phi}\right]$. Thus we get the correct sign in

$$
f e^{2 \phi} D^{-2}=f\left(e^{-\phi / 2} D e^{-\phi / 2}\right)^{-2}+\frac{1}{2} f\left[D, e^{\phi}\right]\left[D, e^{\phi}\right]^{*} D^{-4} .
$$

\section{Acknowledgment}

The research of A. Chamseddine is supported in part by the National Science Foundation under Grant No. Phys-0313416.

\section{References}

[1] S. Coleman, Dilatations in Aspects of Symmetry, Cambridge University Press, 1985, page 67.

[2] W. Buchmüller and N. Dragon, Phys. Lett. B 195 (1987) 417. 
[3] W. Buchmüller and C. Busch, Nucl. Phys. Proc. Supp. B 18 (1990) 295.

[4] R. Holman, E. Kolb, S. Vadas and Y. Wang, Phys. Rev. D43 (1991) 3833.

[5] A. Connes and J. Lott, Nucl. Phys. B 349 (1991) 71.

[6] A. H. Chamseddine, G. Felder and J. Fröhlich, Comm. Math. Phys. 155, 109 (1993).

[7] A. H. Chamseddine, J. Fröhlich and O. Grandjean, J. Math. Phys. 36 (1995) 6255.

[8] A. Chamseddine and J. Fröhlich, Phys. Lett. B 314 (1993) 308.

[9] L. Randall and R. Sundrum, Phys. Rev. Lett. 83 (1999) 3370.

[10] F. Lizzi, G. Mangano and G. Miele, Mod. Phys. Lett. A16 (2001) 1.

[11] A. H. Chamseddine and A. Connes, Phys. Rev. Lett. 77 (1996) 4868; Comm. Math. Phys. 186 (1997) 731.

[12] D. Kastler, Lect. Notes. Phys. 543, (2000) 131-230.

[13] P. Gilkey, Invariance Theory, the Heat Equation and the Atiyah-Singer Index Theorem, Wilmington, Publish or Perish, 1984.

[14] S. Coleman and E. Weinberg, Phys. Rev. D7, (1973) 1888.

[15] W. Buchmüller and N. Dragon, Phys. Lett. B 207 (1988) 292.

[16] Y. Ng and H. van Dam, Phys. Rev. Lett. 65 (1990) 1972.

[17] W. Buchmüller and N. Dragon, Phys. Lett. B 223 (1987) 313.

[18] Y. Ng, Int. J. Mod. Phys. D1 (1992) 145.

[19] A. Connes, J. Math. Phys. 41 (2000) 3832. 